\title{
Growth charts for children with Ellis-van Creveld syndrome
}

\author{
Sabine Verbeek • Paul H. C. Eilers • Kate Lawrence • \\ Raoul C. M. Hennekam • Florens G. A. Versteegh
}

Received: 6 July 2010 / Accepted: 6 August 2010 / Published online: 10 September 2010

(C) The Author(s) 2010. This article is published with open access at Springerlink.com

\begin{abstract}
Ellis-van Creveld (EvC) syndrome is a congenital malformation syndrome with marked growth retardation. In this study, specific growth charts for EvC patients were derived to allow better follow-up of growth and earlier detection of growth patterns unusual for EvC. With the use of 235 observations of $101 \mathrm{EvC}$ patients (49 males, 52 females), growth charts for males and females from 0 to 20 years of age were derived. Longitudinal and cross-sectional data were collected from an earlier review of growth data in $\mathrm{EvC}$, a database of EvC patients, and from recent literature. To model the growth charts, the GAMLSS package for the R statistical program was used. Height of EvC patients was compared to healthy children using Dutch growth charts. Data are presented both on a scale for age and on a scale for the square root of age. Compared to healthy Dutch children, mean
\end{abstract}

S. Verbeek $(\bowtie) \cdot$ F. G. A. Versteegh

Department of Pediatrics, Groene Hart Ziekenhuis,

P.O. Box 1098, Gouda, The Netherlands

e-mail: sabineverbeek@hotmail.com

F. G. A. Versteegh

e-mail: florens.versteegh@ghz.nl

P. H. C. Eilers

Department of Medical Statistics, Erasmus Medical Centre,

Rotterdam, The Netherlands

\section{K. Lawrence}

Springburn Health Centre,

Glasgow, Scotland, UK

R. C. M. Hennekam

Institute for Child Health,

Great Ormond Street Hospital for Children, UCL,

London, UK

R. C. M. Hennekam

Department of Pediatrics, Academic Medical Center,

Amsterdam, The Netherlands height standard deviation score values for male and female EvC patients were -3.1 and -3.0 , respectively. The present growth charts should be useful in the follow-up of $\mathrm{EvC}$ patients. Most importantly, early detection of growth hormone deficiency, known to occur in $\mathrm{EvC}$, will be facilitated.

Keywords Growth · Body height · Ellis-van Creveld syndrome $\cdot$ Growth charts

\section{Introduction}

Ellis-van Creveld syndrome (EvC) (OMIM \# 225500) is a skeletal dysplasia characterized by short limbs, short ribs, postaxial polydactyly, and dysplastic nails and teeth. Sixty percent of affected individuals have a congenital cardiac defect, most commonly an atrial septum defect $[3,4]$. The syndrome is named after the physicians Ellis and van Creveld, who described the first cases in 1940 [4]. EvC is uncommon, the incidence in the general population being 7 per 1,000,000 live births [15]. In the Old Amish population, the incidence is much higher, with 5 per 1,000 live births and as many as $13 \%$ of the Amish people are carrier of the mutation [8]. The disorder follows an autosomal recessive pattern of inheritance. Mutations in two genes (EVC and $E V C 2)$ on chromosome 4 p16 have been identified to be responsible for the EVC phenotype [12].

After birth, children with EVC present with short limbs. The shortening of the limbs increases from proximal to distal, with the most pronounced shortening of the distal interphalangeal bones. A recent study showed that growth hormone deficiency can play a role in the retarded growth in at least some EvC patients [19]. The same study showed variation of the growth retardation from -2.0 to -4.5 standard deviation score (SDS) of average height. The 
authors argued that growth charts specifically for $\mathrm{EvC}$ patients would be useful, as has been found for other entities that go along marked growth retardation, such as Down syndrome, Turner syndrome, and achondroplasia [5, 10, 16, 18]. Such dedicated growth charts allow better follow-up of growth and earlier detection of growth patterns unusual for the entity involved. This urged us to create growth charts specifically for children with EvC.

\section{Methods}

Patients

Growth data of $101 \mathrm{EvC}$ patients were available for analysis. Data were gathered from different sources. For our previous study [19], we gathered measurements of 80 patients. From these studies, four patients were known to receive therapy for growth hormone deficiency and were therefore excluded. All cases from literature that were molecularly proven were added; patients of whom molecular results were not available were included if the diagnosis was thought to be right by an experienced clinical geneticist. One hundred and three measurements of 76 patients from these studies were used for analysis. Secondly, we used the database of an EvC website (www.ellisvancreveld.co.uk). This database provided 126 data points of 19 subjects with EvC. In all latter patients, the diagnosis was molecularly proven. There was no overlap between subjects of this database and subjects from our previous studies. Thirdly, we added data from literature published after our 2007 study was concluded $[1,3,17]$. This provided us eight measurements of six patients. In total, 229 usable data were available of 101 patients - 106 data points of 49 males and 123 data points of 52 females.

\section{Statistical analysis}

To model the growth charts, the GAMLSS package for the $\mathrm{R}$ statistical program (R Development Core Team, 2009) was used [13]. GAMLSS models the conditional distribution of height for a given age by means of three curves: one for the mean, one for the (logarithm of the) standard deviation, and one for a power transformation to normality.

To find the best balance between complexity and fit to the data, transformation to normality was not performed, and linear trends (in the square root of age) were taken for the mean curve and the logarithm of the standard deviation. With this model, percentile curves were computed.

To compare height of $\mathrm{EvC}$ patients with height in healthy children, we presented the data in digital Dutch growth curves [7].

\section{Results}

A total of 229 measurements on 101 subjects were used for the analysis. Of 29 subjects, we gathered more than 1, and of 12 subjects, we gathered more than 5 measurements (Table 1). Of the 52 female subjects, 48 were aged between 0 and 20 years and 4 subjects were above 20 years. We assumed height of these patients would be comparable with 20-year-old patients and therefore treated these data points as such. Indeed, if leaving these data points outside the analysis, the growth curves did not change. Of the 49 male subjects, 42 were aged between 0 and 18 years and 6 were above 20 years of age. Again, we treated the data of these patients as if they were 20-year-old patients. As in the females, the growth curve did not change if these data points were left out of the analysis.

Data from the literature were published between 1940 and 2009. Ethnicity differed since data were gathered from all over the world. Most of the patients $(n=60)$ were of Caucasian descent, 15 were Hispanic, 24 patients had other ethnic backgrounds (African-American, Jewish, Turkish, Chinese, Mongolian, Japanese, Indian), and of 2 patients from literature, the ethnicity was not known. Consanguinity of parents in patients collected from the literature was mostly unknown to us. In the UK database, none of the parents was consanguineous.

The growth charts were derived for males from 0 to 20 years of age (Figs. 1, 2, and 5) and females from 0 to 20 (Figs. 3, 4, and 6). Transformation of age to a different scale, by taking square roots, led to almost linear curves (Figs. 1 and 3). Figures 2 and 4 show growth charts for boys and girls, respectively, with all the collected data points. Figures 5 and 6 show the same growth charts without these data points, to allow use for patient care purposes. Curves are made for the specific age group. The statistical program used here does not make it possible to extrapolate data points to adulthood.

Height SDS values for EvC patients are shown on Dutch growth charts for boys (Fig. 7) and girls (Fig. 8) of 0

Table 1 Patient characteristics

\begin{tabular}{lcc}
\hline Age group (years) & $\begin{array}{l}\text { Male data points } \\
(n) / \text { male patients } \\
\text { in age group }(n)\end{array}$ & $\begin{array}{l}\text { Female data points } \\
(n) / \text { female patients } \\
\text { in age group }(n)\end{array}$ \\
\hline $0-1$ & $36 / 18$ & $63 / 28$ \\
$1-5$ & $38 / 15$ & $34 / 15$ \\
$5-12$ & $16 / 13$ & $13 / 8$ \\
$12-20$ & $10 / 10$ & $9 / 8$ \\
$>20$ & $6 / 6$ & $4 / 4$ \\
Total & $106 / 62$ & $123 / 63$ \\
\hline
\end{tabular}

Data points of male and female patients per age group 


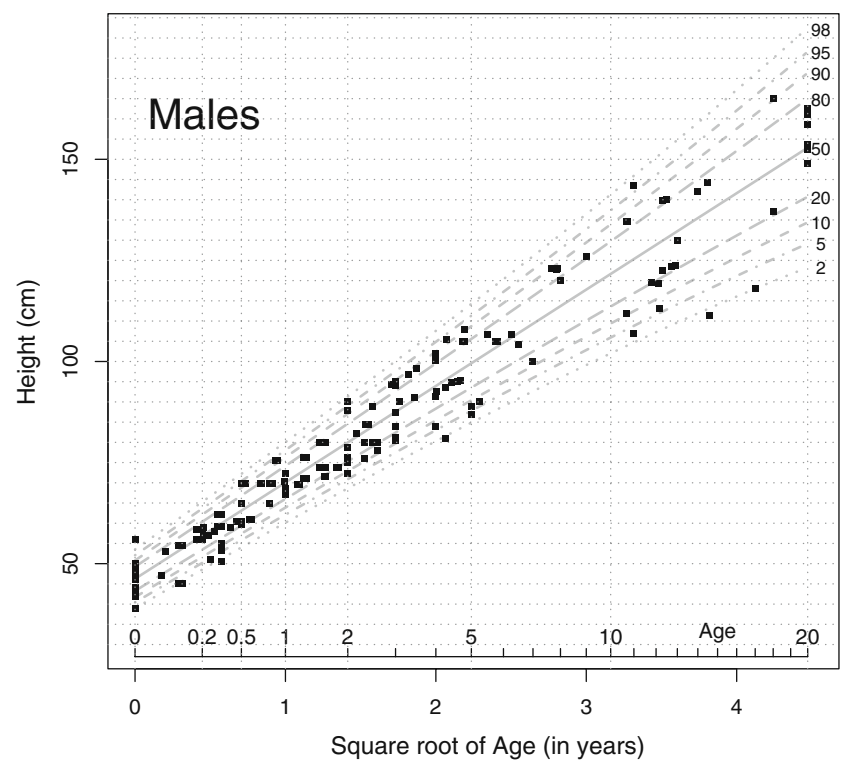

Fig. 1 Growth chart for boys from 0 to 20 years of age with Ellis-van Creveld syndrome $(n=49)$. Curves presented as height (centimeters) versus square root of age (years)

21 years. SDS values vary between +1.4 and -8.6 , with a mean of $-3.1 \mathrm{SD}$ for male $\mathrm{EvC}$ patients and $-3.0 \mathrm{SD}$ for female EvC patients [7].

\section{Discussion}

Syndrome-specific growth charts have been developed for several syndromes, such as Down syndrome, Turner syndrome, and Williams syndrome $[5,10,16]$. These charts

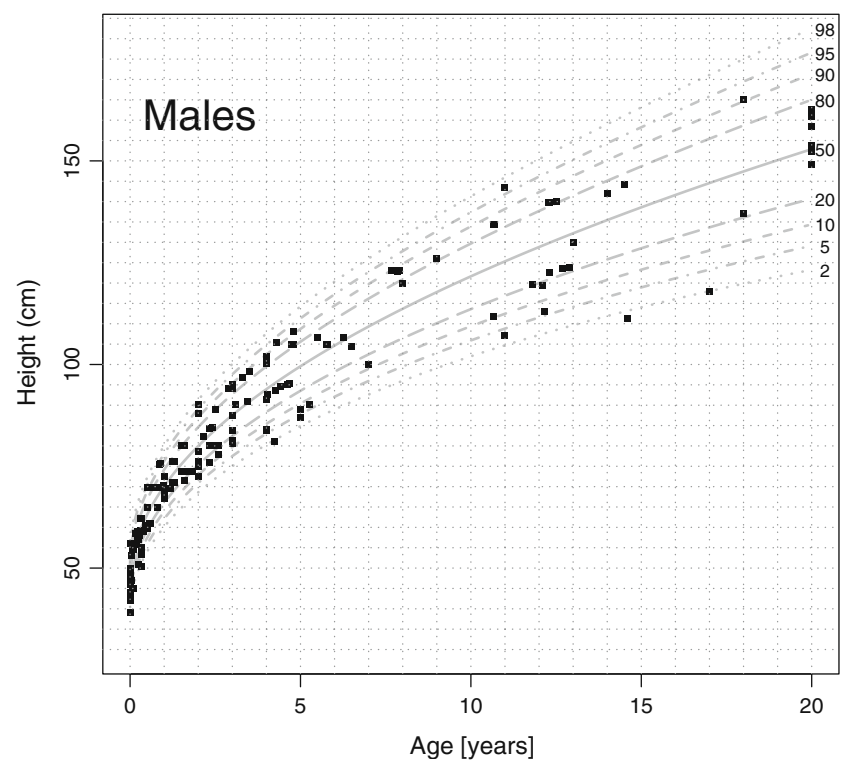

Fig. 2 Growth chart for boys from 0 to 20 years of age with Ellis-van Creveld syndrome $(n=49)$. Curves presented as height (centimeters) versus age (years)

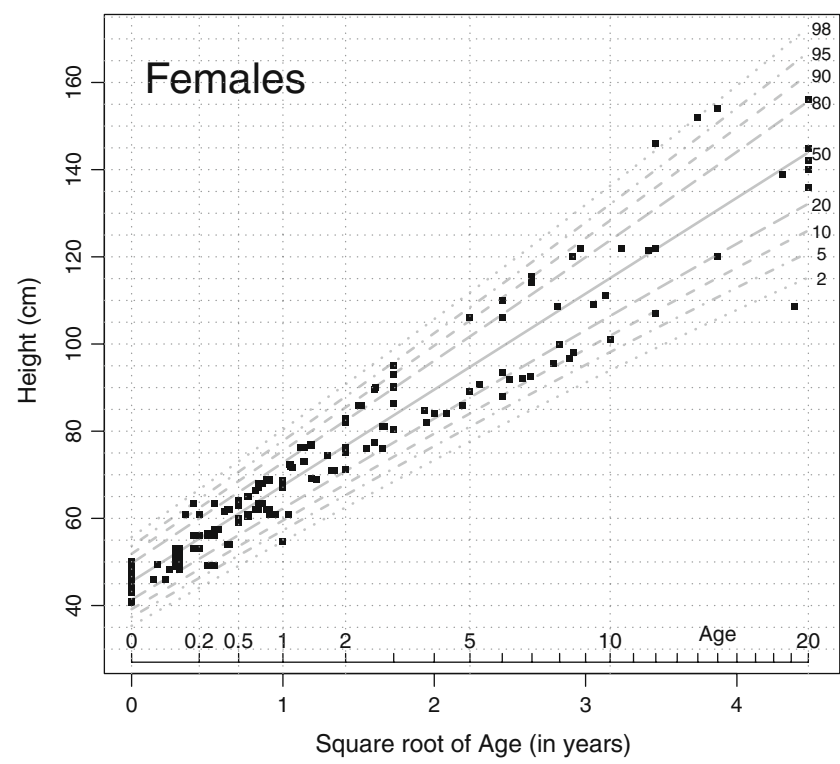

Fig. 3 Growth chart for girls from 0 to 20 years of age with Ellis-van Creveld syndrome $(n=52)$. Curves presented as height (centimeters) versus square root of age (years)

have proven to be a helpful tool in the medical care of these children. Growth in EvC is known to be impaired, with an estimated deviation of -2.0 to -4.5 from standard growth [19]. Until recently, it was assumed to be explainable merely because of the skeletal dysplasia. However, growth hormone deficiency has been reported in patients with $\mathrm{EvC}$ and its frequency remains uncertain [19]. This may aggravate the growth retardation. To detect growth hormone deficiency and other disorders that cause additional growth deviation in EvC patients, specific growth charts for

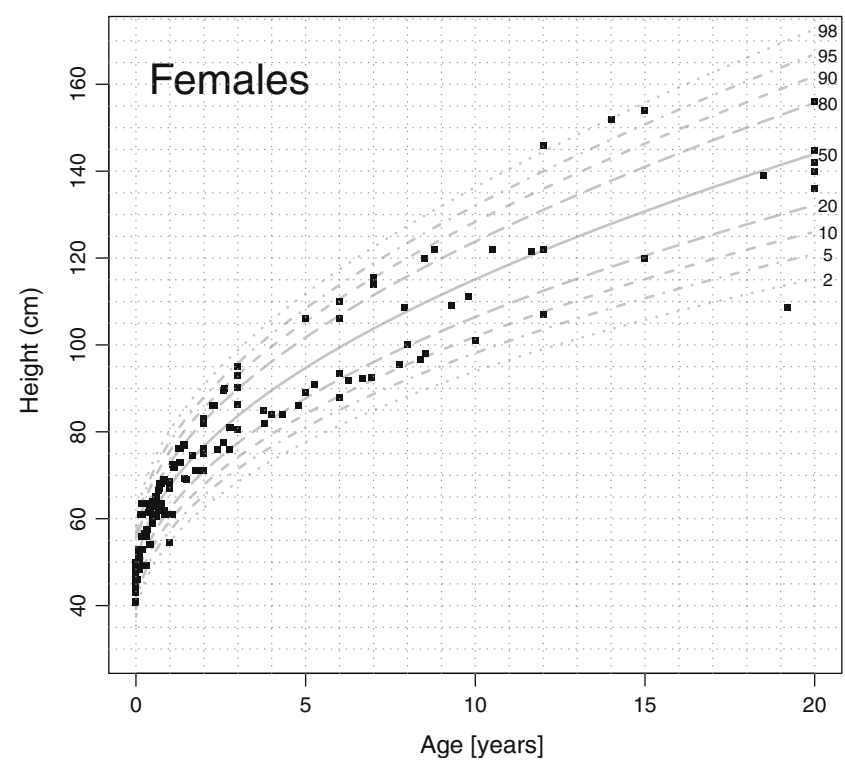

Fig. 4 Growth chart for girls from 0 to 20 years of age with Ellis-van Creveld syndrome $(n=52)$. Curves presented as height (centimeters) versus age (years) 


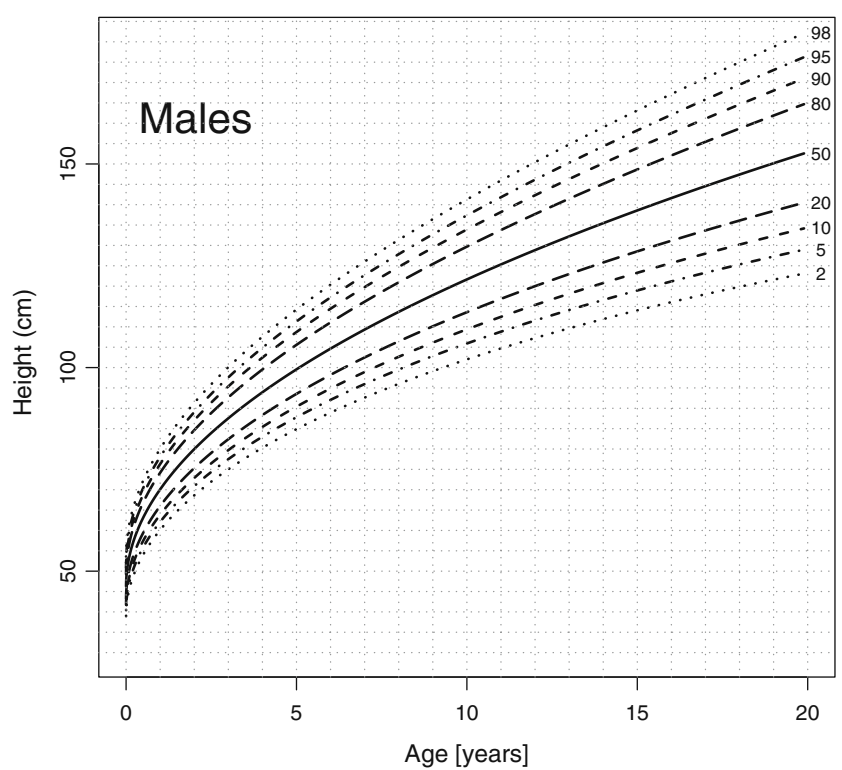

Fig. 5 Growth chart for boys from 0 to 20 years of age with Ellis-van Creveld syndrome

children with $\mathrm{EvC}$ should be of great value. We present here growth charts for males and females with EvC.

Since the data set was small, a simple model was aimed for. Transformation of age to a different scale, by taking square roots, led to almost linear curves. Furthermore, it spreads out the data for the younger age group, which makes it more clarifying.

We treated the data as cross-sectional although a number of measurements come from the same individuals. There is no provision in the statistical program GAMLSS for grouped data. The alternative would be to select one

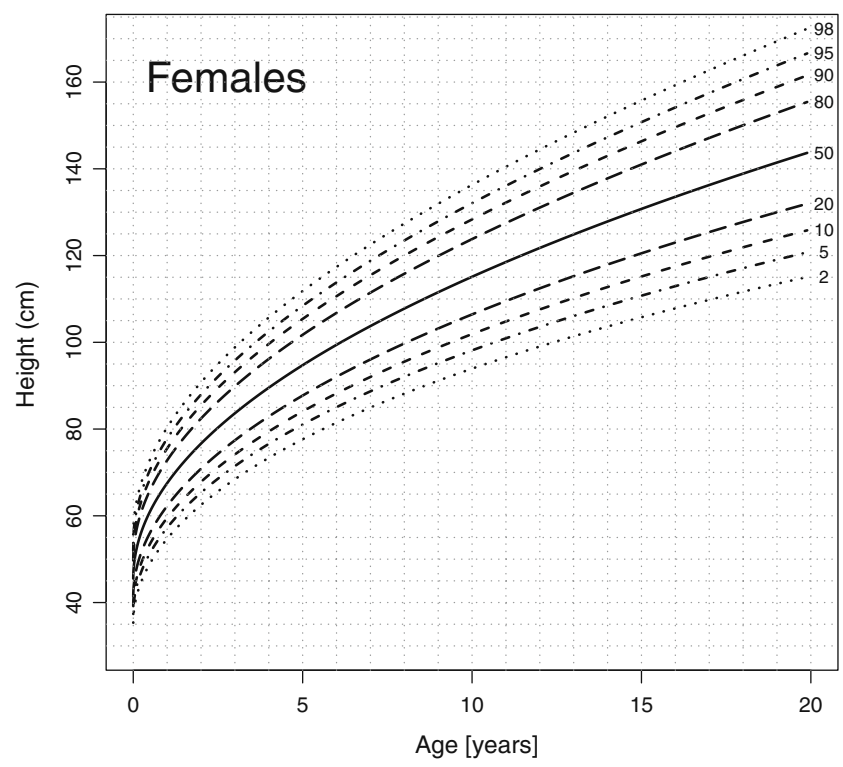

Fig. 6 Growth chart for girls from 0 to 20 years of age with Ellis-van Creveld syndrome

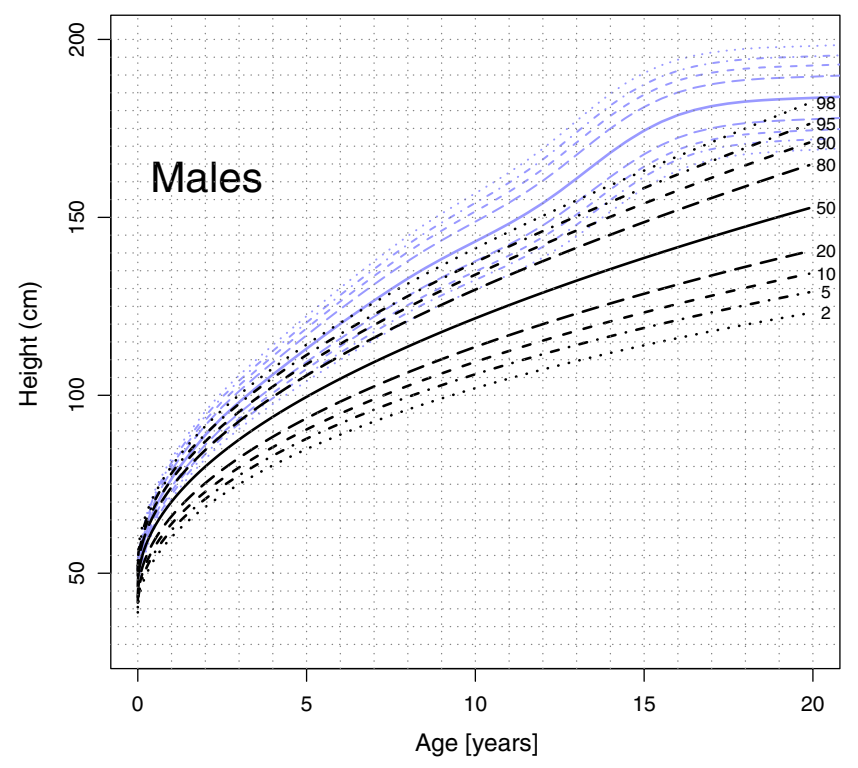

Fig. 7 Dutch growth charts for boys (0-21 years, blue line) with height SDS of boys with Ellis-van Creveld syndrome (0-20 years, dotted line)

measurement per individual or leave repeated measurements out completely. Both choices were unattractive. Because our analysis is mainly descriptive, we believe that little harm was done by using all data.

To compare growth of EvC patients to healthy children, data were shown in Dutch growth charts. Mean growth deviation was $-3.1 \mathrm{SD}$ for male patients and $-3.0 \mathrm{SD}$ for female patients, comparable to our previous study [19].

A drawback of the present study is the possible influence of the secular trend in growth. Data were gathered from

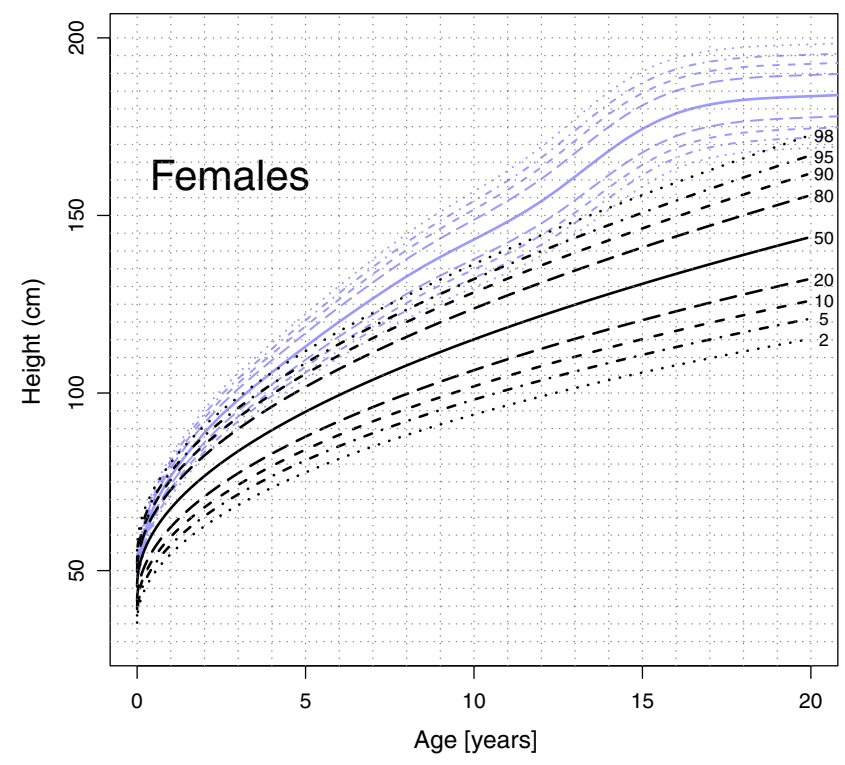

Fig. 8 Dutch growth charts for girls (0-21 years, blue line) with height SDS of girls with Ellis-van Creveld syndrome (0-20 years, dotted line) 
publications between 1940 and 2009. Nowadays, boys and girls are taller than in the 1940s [10, 14]. Although the proportion of data from more than 30 years ago is relatively small (28 data points), the influence of the secular trend cannot be ruled out. Another drawback is that data were gathered from all over the world. Consequently, the here presented growth charts represent different cultures and ethnicities, which can differ greatly $[2,9,11]$. We reasoned, however, that in EvC the growth retardation would be that extreme that this would easily outweigh the ethnic growth differences and we therefore used all data.

When compared to regular growth charts, the here presented growth charts differ in form. It seems that growth does not slow down to a complete horizontal curve after puberty. The reason for this is the statistical approach used in this study. Since the data set was small, the formation of a splined curve was not possible. Regular growth charts show an S-shaped or "splined" curve. These curves flatten in adolescence, leading to an end height or target height. The here presented growth charts are developed with the use of another statistical approach, used for smaller databases [13]. This method does not allow extrapolation of the curve. Hence, the here presented growth charts can only be used in a specific age group. More research with a larger database would be necessary to develop growth charts with a splined curve. ${ }^{1}$

Open Access This article is distributed under the terms of the Creative Commons Attribution Noncommercial License which permits any noncommercial use, distribution, and reproduction in any medium, provided the original author(s) and source are credited.

\section{References}

1. Abeles AI, Tobias JD (2008) Anaesthetic implications of Ellisvan Creveld syndrome. J Clin Anesth 20:618-621

2. de Onis M, Onyango AW, Borghi E et al (2007) Development of a WHO growth reference for school-aged children and adolescents. Bull World Health Organ 85:660-667

3. Digilio MC, Marino B, Ammirati A et al (1999) Cardiac malformations in patients with oral-facial-skeletal syndromes: clinical similarities with heterotaxia. Am J Med Genet 84:350-356
4. Ellis RW, Van Creveld SA (1940) Syndrome characterized by ectodermal dysplasia, polydactyly, chondro-dysplasia and congenital morbius cordis: report of three cases. Arch Dis Child 15:65-69

5. Gawlik A, Gawlik T, Augustyn M et al (2006) Validation of growth charts for girls with Turner syndrome. Int J Clin Pract 60:150-155

6. Gohlke B, Woelfle J (2009) Growth and puberty in German children: is there still a positive secular trend? Dtsch Arztebl Int 106:377-382

7. Growth Analyser Junior (2006)

8. Ide SE, Ortiz RI, Francomano CA, Polymeropoulos MH (1996) Exclusion of the MSX1 homeobox gene as the gene for the Ellis-van Creveld syndrome in the Amish. J Human Genet 98:572-575

9. Lejarragaa H, del Pinoa M, Fanoa V et al (2009) Growth references for weight and height for Argentinian girls and boys from birth to maturity. Incorporation of data from the World Health Organisation from birth to 2 years and calculation of new percentiles and LMS values. Arch Argent Pediatr 107:126-133

10. Martin ND, Smith WR, Cole TJ, Preece MA (2007) New height, weight and head circumference charts for British children with Williams syndrome. Arch Dis Child 92:598-601

11. Ogden CL, Kuczmarski RJ, Flegal KM et al (2002) Centers for disease control and prevention 2000 growth charts for the United States: improvements to the 1977 National Center for Health Statistics version. Pediatrics 109:141-142

12. Polymeropoulos MH, Ide SE, Wright M et al (1996) The gene for the Ellis van Creveld syndrome is located on $4 \mathrm{p} 16$. Genomics 35:1-5

13. Rigby RA, Stasinopoulos DM (2005) Generalized additive models for location, scale and shape. Appl Statist 54:507-554

14. Roelants M, Hauspie R, Hoppenbrouwers K (2009) References for growth and pubertal development from birth to 21 years in Flanders, Belgium. Ann Hum Biol 7:1-15

15. Stoll C, Dott B, Roth MP, Alembik Y (1989) Birth prevalence rates of skeletal dysplasias. Clin Genet 35:88-92

16. Styles M, Cole T, Dennis J, Preece M (2002) New cross sectional stature, weight, and head circumference references for Down's syndrome in the UK and Republic of Ireland. Arch Dis Child 87:104-108

17. Thapa R, Mukhopadhyay M, Bhattacharya A (2008) Discordance for Ellis-van Creveld syndrome in twins. Singapore Med J 49:369-371

18. Trotter TL, Hall JG, Committee on Genetics (2005) Health supervision for children with achondroplasia. Pediatrics 116:771783

19. Versteegh FGA, Buma SA, Costin G et al (2007) Growth hormone analysis and treatment in Ellis-van Creveld syndrome. Am J Med Genet 143A:2113-2121

\footnotetext{
$\overline{{ }^{1} \text { Growth data }}$ of children with proven $\mathrm{EvC}$ can be sent to evc@crydee.plus.com.
} 